\title{
Quelques Nématodes parasites de Blattes
}

\section{à Madagascar}

\author{
par D. VAN WAEREBEKE \\ Centre O.R.S.T.O.M. de Tananarive (Madagascar)
}

\begin{abstract}
Résumé
Trois nouvelles espèces de Thelastomatidae parasites de blattes sont décrites: Protrellus rasolofi, Protrellus behorefi et Thelastoma madecassa, ainsi que la forme Malgache de Thelastoma pachyjuli (Parona 1896), Travassos 1929.
\end{abstract}

\section{Summary}

Among malagasian Thelastomatidae parasitic of cokroaches 3 new species are described and figured: Protrellus rasolofi, Protrellus behorefi and Thelastoma madecassa.

La plupart des espèces de Blattes recensées à Madagascar (une centaine environ) sont endémiques. Jusqu'à présent, aucune étude n'a été entreprise dans ce pays sur les nombreux parasites et commensaux du tube digestif. Nous nous sommes intéressés ici à quatre espèces de Thelastomatidae qui font l'objet de descriptions détaillées. Les parasites de la cavité générale sont moins fréquents. L'étude du parasitisme de la blatte Gromphadorhina portentosa, Schaum, par des tachinaires, des larves de mermis et des stades intermédiaires de Nématodes parasites de vertébrés sera abordée ultérieurement. 


\section{Quatre Oxyures associés aux Blattes à Madagascar}

\section{$1^{\circ}$ Protrellus rasolofi n. sp.}

\section{DESCRIPTION DE LA FEMELLE:}

Longueur du corps : $5600-7660 \mu$.

Largeur du corps : $159-179 \mu ; a * 34-42$.

Longueur de l'œsophage: $450-478 \mu ; b$ 12-16,5.

Longueur de la queue: $460-505 \mu ; c 18,5-15,7$.

Distance de la vulve à l'apex : $260-325 \mu ; \mathrm{V} 4,1-5,4 \%$.

$n: 11$.

Les mesures suivantes sont relatives à l'holotype de longueur $6950 \mu$.

Le corps de la femelle est long. La cuticule n'est striée qu'au niveau du corpus. Le premier anneau a une forme caractéristique (plus ou moins trapézoïdale, longueur $20 \mu$ ). Les anneaux suivants ont une longueur croissante (de 10 à $20 \mu$ ) jusqu'à la vulve. Puis les stries s'estompent et leur écartement diminue. Il n'y a pas d'aile latérale. En vue apicale, la tête (diamètre $29 \mu$ ) présente huit papilles céphaliques bien visibles et deux amphides assez réduites. La paroi externe du stoma forme un anneau dont le sommet est interrompu par trois encoches semi-circulaires. Le corpus (longueur $312 \mu$ ) est composé d'un procorpus cylindrique (diamètre $26 \mu$ ) et d'un métacorpus dilaté (diamètre $59 \mu$ ). L'isthme (diamètre $16 \mu$ ) n'est qu'un court étranglement entre le corpus et le bulbe (longueur isthme plus bulbe $130 \mu$, diamètre du bulbe $104 \mu$ ). L'anneau nerveux est à $180 \mu$ de l'apex et le pore excréteur est à $29 \mu$ de la vulve $104 \mu$. L'orifice du pore excréteur est situé sous un repli cuticulaire en forme de languette. La partie antérieure de l'intestin moyen est élargie en un cardia dont la paroi forme des replis longitudinaux qui divisent partiellement la cavité intestinale. L'appareil. génital est didelphe. L'ovariole correspondant à l'ovaire antérieur prend son origine dans la partie postérieure du corps. Il est replié deux fois sur lui-méme, la première fois, près de la queue et la seconde fois à mi-corps ; il se prolonge alors par un oviducte bien développé. L'ovariole correspondant à l'ovaire postérieur a une position symétrique par rapport au premier et prend son origine au milieu du corps. Les deux oviductes se rejoignent aux deux tiers de la longueur du corps où ils fusionnent. L'utérus, unique, volumineux peut contenir plus de 500 œufs. Il s'amincit dans sa partie antérieure où les œufs sont expulsés un à un. La vulve légèrement saillante est située au niveau du métacorpus. Les œufs (longueur 65-73 $\mu$, largeur 38-45 $\mu$ ) sont aplatis sur une face. La queue est longue et conique ; son extrémité est effilée.

DESCRIPTION DU MÂLE :

Longueur du corps : $755 \mu$.

Largeur du corps: $58,5 \mu ; a 12,4$.

* $a, b, c, \mathrm{~V}$ : rapports de De Man. 


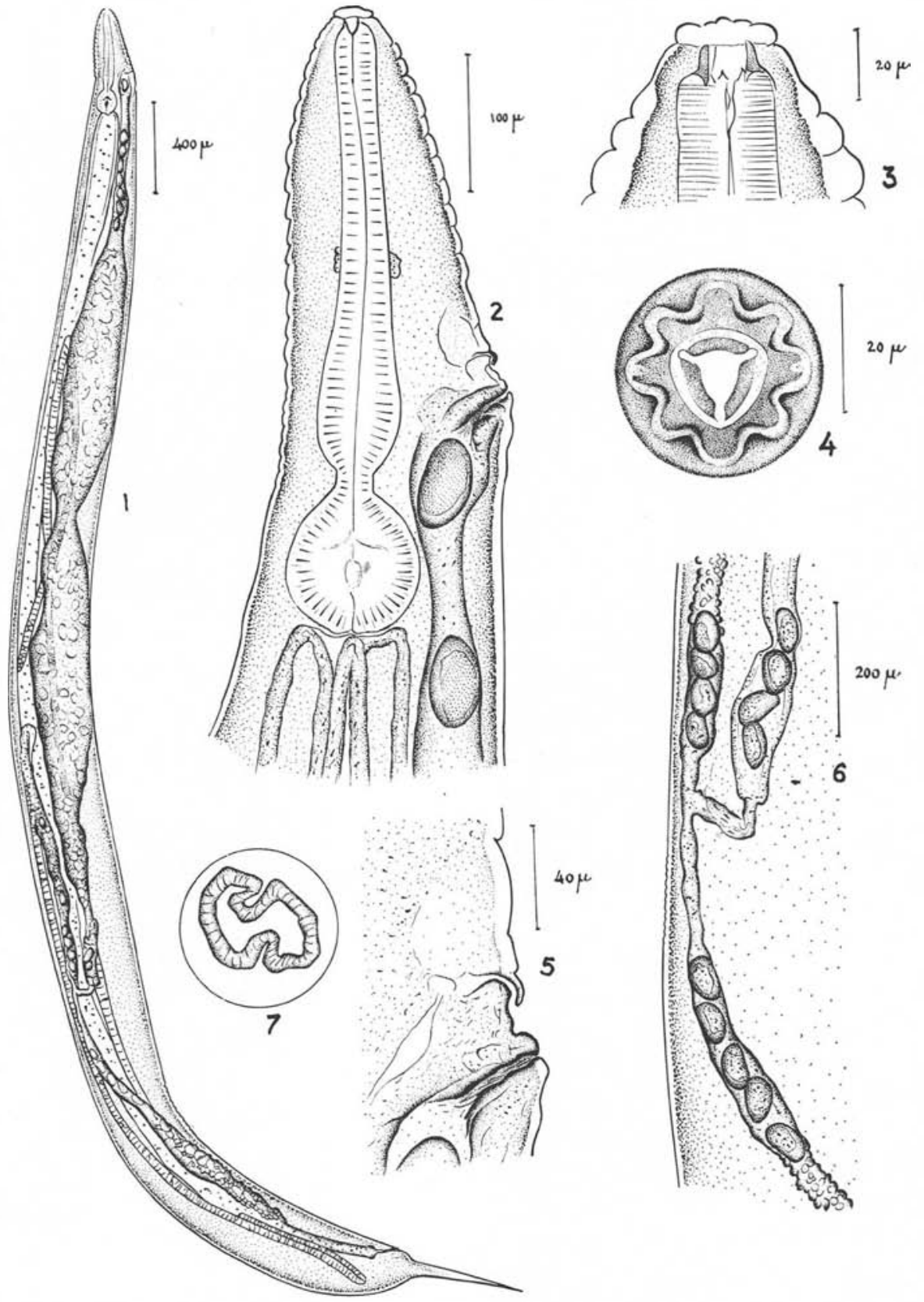

PL. I et II. - Protrellus rasolofi n. sp.

A) La femelle: 1. Vue d'ensemble; 2. La partie antérieure du tube digestif; 3. La tête, vue latéralè ; 4. La têté, vue apicale; 5 . La vulve et le pore excréteur; 6. Détail de l'appareil génital; 7. Coupe transversale de la partie antérieure de l'intestin moyen.

Annales de Parasitologie, humaine et comparée (Paris), t. 44, 1969, $\mathrm{n}^{\circ} 6$ 

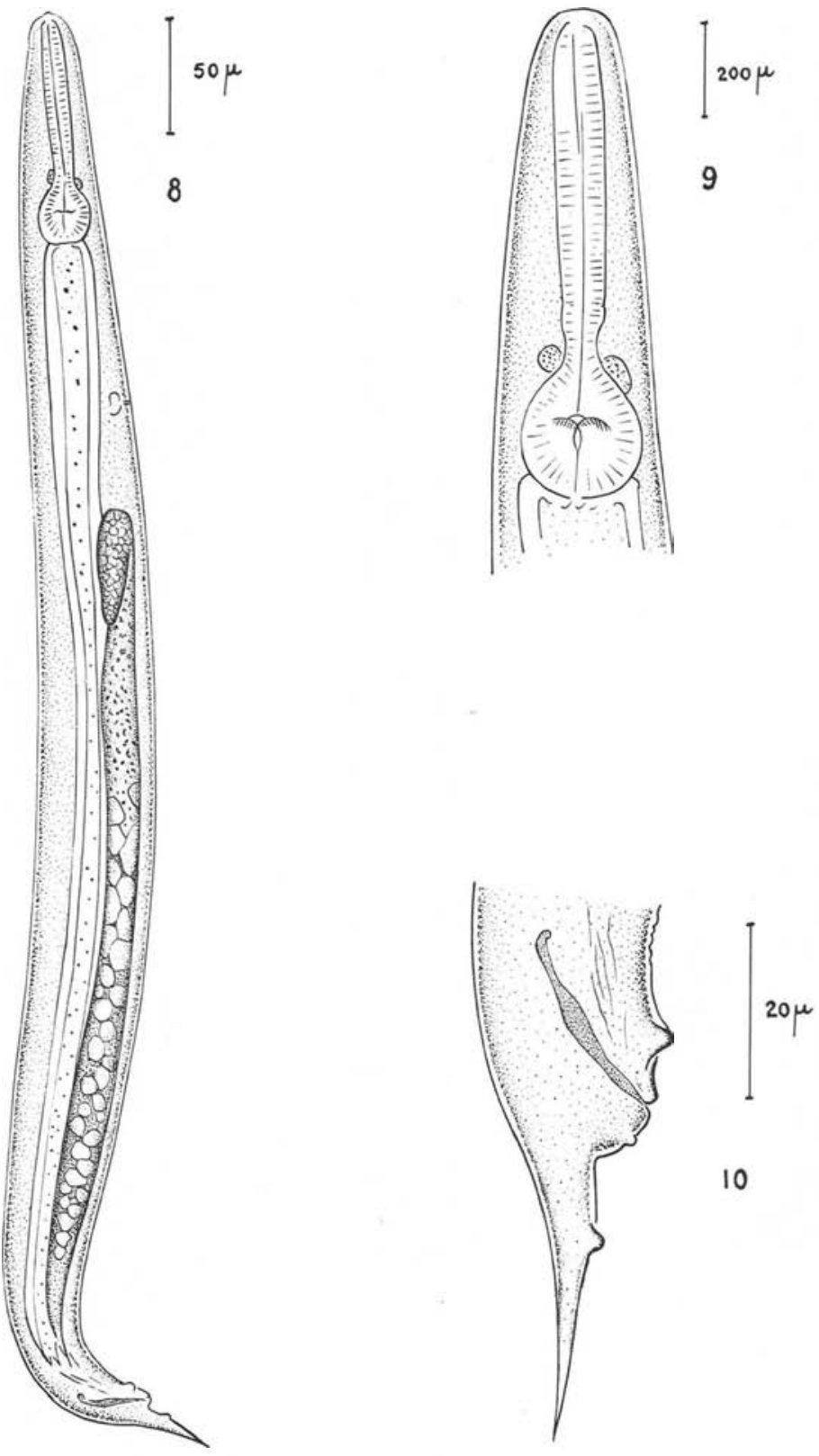

B) Le mâle : 8. Vue d'ensemble ; 9. L'œsophage; 10. Le cône génital et la queue. 
Longueur de l'œsophage: $102 \mu ; b$ 7,2.

Longueur de la queue: $39 \mu$; 20 .

Ces mesures sont celles du seul mâle que nous ayons trouvé. On distingue difficilement les stries sur la cuticule. Il n'y a pas d'aile latérale. L'œsophage est relativement petit. Le corpus est cylindrique (longueur 67,5 $\mu$, diamètre $13 \mu$ ). L'anneau nerveux est situé à la limite entre l'isthme (longueur 12,5 $\mu$, diamètre 8,3 $\mu$ ) et le bulbe (longueur $31 \mu$, diamètre $26 \mu$ ). L'intestin moyen est légèrement élargi dans sa partie antérieure. Le pore excréteur est très en arrière du bulbe, à $160 \mu$ de l'apex. Le testicule est replié sur lui-même. Le spicule mesure environ $21 \mu$ de long. La queue est conique. La première paire de papille, préanale, est bien développée, la deuxième postnatale, est réduite. La troisième paire est située à $24 \mu$ de l'extrémité caudale.

Habitat et provenANCE : Intestin postérieur de Gymnonyx grandidieri Saussure 1895, Forêt de l'Ankarafantsika.

Quelques Éléments de DiAgnose: Vulve de la femelle au niveau du métacorpus. Métacorpus large. Paroi de la partie antérieure de l'intestin moyen plissée longitudinalement. Queue pointue en forme de cône allongé. Eufs aplatis sur une face.

\section{Discussion.}

Les oxyures dont la vulve est antérieure à la base du bulbe forment un groupe original. Parmi ceux-ci, Basir (1956) distingue quatre genres, trois d'entre eux comprenant une seule espèce et le genre Protrellus Cobb 1920, sept espèces. Les genres Protrelloides Chitwood, 1932, et Protrelleta Chitwood, 1932, sont respectivement caractérisés par un œsophage long et fin et des papilles céphaliques digitiformes. Basir (1932) crée le genre Linstowiella pour distinguer l'espèce Linstowiella lanceolata (Linstow 1883), Travassos, 1929, dont la queue est longue et filiforme, des espèces au genre Protrellus (queue conique très réduite). L'espèce décrite ci-dessus se place entre les deux genres avec une queue conique et effilée (comme Protrellina gurri Dale 1966). Nous l'avons placée dans le genre Protrellus où elle se distingue par les caractères de l'œsophage de l'intestin moyen, de la queue, etc...

\section{Remarque.}

KLoss (1961-1966) ne reconnaît pas le genre Protrellus Cobb, 1920, et décrit plusieurs espèces qu'il attribue au genre Protrellina ( $P$. eurycotesi, $P$. ischnopterae, $P$. ituana) créé par Chitwood en 1932 (ce dernier auteur envisageant lui-même la synonymie de Protrellina avec Protrellus). Il décrit, en outre, une nouvelle espèce du genre Protrelleta, P. labrumspinosa et crée les nouveaux genres Aglaopterixia et Napolitana, isolant ainsi Aglaopterixia manni (Chitwood 1932) et Napolitana galebi (Schwenk 1926) du genre Protrellus. 


\section{$2^{\circ}$ Protrellus behorefi n. sp.}

DESCRIPTION DE LA FEMELLE:

Longueur du corps : $3440-5650 \mu$.

Largeur du corps : 182-338 $\mu$; a 16-22.

Longueur de l'œsophage: 390-432 $\mu ; b$ 10-14.

Longueur de la queue: $135-182 \mu ; c$ 24-36.

Distance de la vulve à l'apex: 192-218 $\mu$; V 3,5-5,9\% .

$n: 24$.

Les mesures qui suivent sont relatives au type de longueur $4280 \mu$.

Le corps est long et à peu près cylindrique. La tête précède un premier anneau plus grand que les suivants (longueur $10 \mu$ ). La cuticule n'est striée qu'au niveau du corpus (distance entre chaque strie variant de 6 à $12 \mu$ ). Il n'y a pas d'aile latérale. La queue est conique et courte.

L'ouverture buccale est arrondie. Les amphides sont bien visibles, contrairement aux huit papilles du cercle céphalique externe, peu proéminentes. On peut aussi apercevoir à un fort grossissement les six papilles céphaliques internes déjà observées par Dale (1966) sur Protrellina gurri. A la base du stoma, l'ouverture œsophagienne triradiée forme trois petites cavités sous les rhabdions. Les trois dents œsophagiennes sont réduites. L'œsophage est formé par le corpus cylindrique (longueur $322 \mu$, diamètre variant de $22 \mu$ près du stoma à $31 \mu$ avant l'isthme), un isthme court (longueur $13 \mu$, diamètre $21 \mu$ ) et le bulbe (diamètre $74 \mu$ ). L'anneau nerveux est à $148 \mu$ de l'apex. L'intestin est légèrement dilaté dans sa partie antérieure.

La vulve, située au niveau du milieu de l'œsophage, est saillante. Le pore excréteur est à $180 \mu$ de l'apex et à $28 \mu$ de la vulve. L'utérus, qui contient un grand nombre d'œufs, descend jusqu'à mi-corps où il se divise en deux segments prolongés par les deux ovarioles repliés une ou deux fois sur eux-mêmes. Les œufs (grand diamètre 72-77 $\mu$, plus petit diamètre 34-40 $\mu$ ) ont une face aplatie ou légèrement concave.

LE MÂLE EST INCONNU.

Hôte: Elliptoblatta antennata Saussure, 1895 (Intestin postérieur).

Localité: Behorefo, près d'Ambatondrazaka.

Quelques ÉlÉments DE DIAGNOSE : femelle à queue courte, conique, Six papilles céphaliques internes et huit papilles céphaliques externes peu proéminentes. Lumière œsophagienne formant trois petites loges sous le stoma. Vulve saillante.

\section{Diseussion.}

Nous n'avons pas relevé de différences notoires avec la description de Protrellus galebi Schwenk, 1926, mais l'imprécision de cette description ne nous permet pas de conclure s'il s'agit d'une même espèce. La nouvelle description faite par Kloss en 

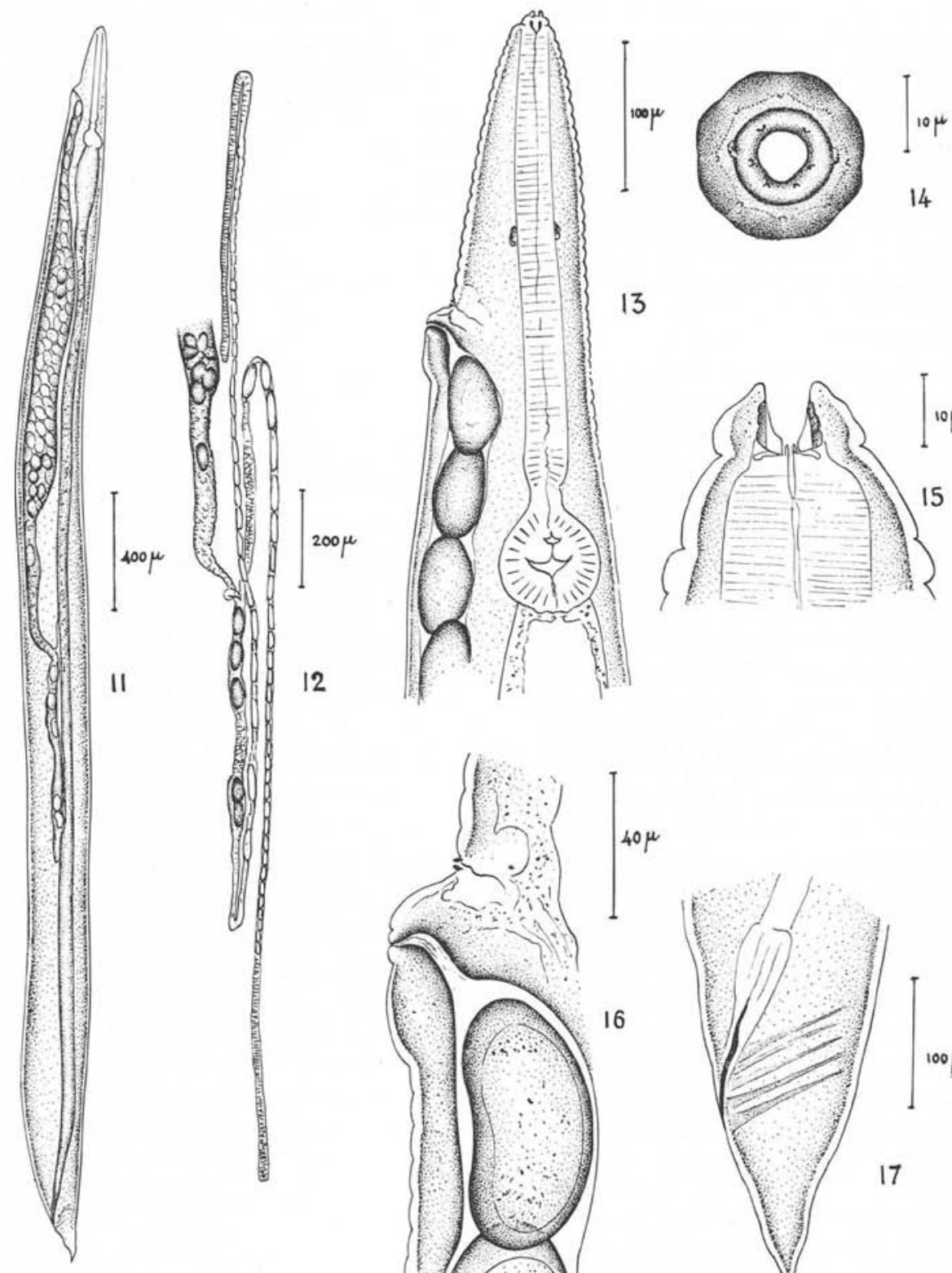

13

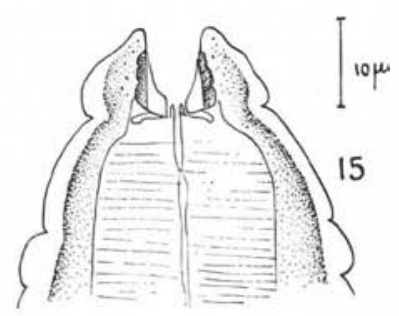

12
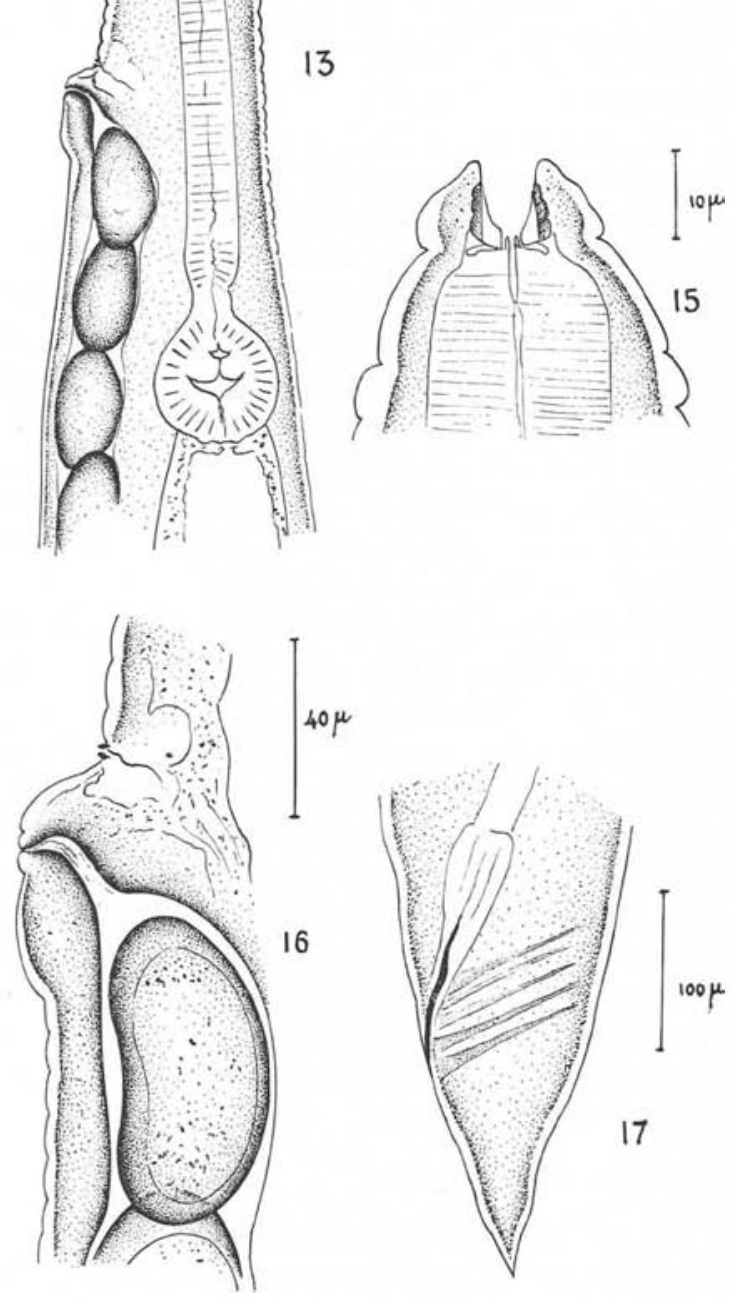

PL. III. - Protrellus behorefi n. sp.

11. Vue d'ensemble; 12. Détail de l'appareil génital; 13. L'œsophage; 14. Vue apicale de la tête; 15. Vue latérale de la tête; 16 . La vulve; 17 . La queue. 
1959 ne nous apporte malheureusement pas les précisions nécessaires (vue apicale de la tête, vue latérale du stoma, détail de la vulve et du pore excréteur ?). Cet auteur signale des irrégularités de la cuticule au niveau de l'œsophage, même chez le Nématode vivant, que nous n'avons jamais observées, et se base sur ce caractère pour créer le genre Napolitana avec l'espèce type unique Napolitana galébi. Nous pensons donc qu'il s'agit d'une espèce distincte de la nôtre, ce qui devrait être confirmé par une étude morphologique détaillée de l'espèce Sud-américaine.

\section{$3^{\circ}$ Thelastoma madecassa n. sp.}

\section{DESCRIPTION DE LA FEMELLE :}

Longueur totale du corps : 1944-2509 $\mu$.

Largeur du corps: $192-260 \mu ; a$ 8,8-10,5.

Longueur de l'œsophage: $354-378 \mu ; b$ 5,2-6,9.

Longueur de la queue: $380-530 \mu ; c$ 4,7-5,4.

Distance de vulve à l'apex : 936-1242 $\mu$; V 47,4-52,5\%.

$n: 6$.

Les mesures suivantes sont relatives à l'holotype de longueur $2250 \mu$.

La tête, petite (hauteur $8 \mu$, diamètre $23 \mu$ ) se prolonge par un premier anneau de dimension supérieure aux autres (longueur $14 \mu$, diamètre $42 \mu$ ). La cuticule est striée sur toute la longueur du corps, les premières stries (distantes de $6 \mu$ ) sont bien marquées, les suivantes s'estompent et sont plus espacées (12 $\mu$ vers la vulve). Il n'y a pas d'aile latérale. Les papilles céphaliques sont larges et peu proéminentes. Les trois dents buccales sont bien visibles. Le stoma a un diamètre de $9 \mu$ et une profondeur de $13 \mu$. L'œsophage est composé d'un corpus à peu près cylindrique (longueur $276 \mu$, diamètre $36 \mu$ ) d'un isthme court (longueur $17 \mu$, diamètre minimum $25 \mu$ ) et d'un bulbe sphérique (diamètre $87 \mu$ ). L'anneau nerveux est situé à la moitié de la longueur du corpus (à $161 \mu$ de l'apex) et le pore excréteur est situé au niveau du bulbe, à $384 \mu$ de l'apex. La partie antérieure de l'intestin forme un cardia bien marqué. L'appareil génital est didelphe. Les lèvres de la vulve ne sont pas saillantes. Le vagin est dirigé vers la tête et est replié sur lui-même avant d'atteindre l'utérus contenant des œufs (48 chez l'holotype) dont le grand diamètre varie entre 47 et $54 \mu$ et le petit diamètre entre 40 et $46 \mu$. Les œufs évoluent dans l'utérus et ceux qui sont prêts à être expulsés renferment des larves déjà bien formées dans lesquelies on distingue l'œsophage et l'intestin. La queue, qui a une longueur égale au cinquième de la longueur du corps, est large (et non filiforme).

\section{MâLE INCONNU.}

HôTE: Elliptoblatta madecassa Saussure, 1895.

Localité : piste de Moramanga à Anosibe, Madagascar.

ElÉments DE diAgNose: La queue est conique et de longueur égale au cinquième de celle du corps. Le pore excréteur est en face du bulbe. La larve est déjà formée dans les œufs qui sont expulsés à l'intérieur. 

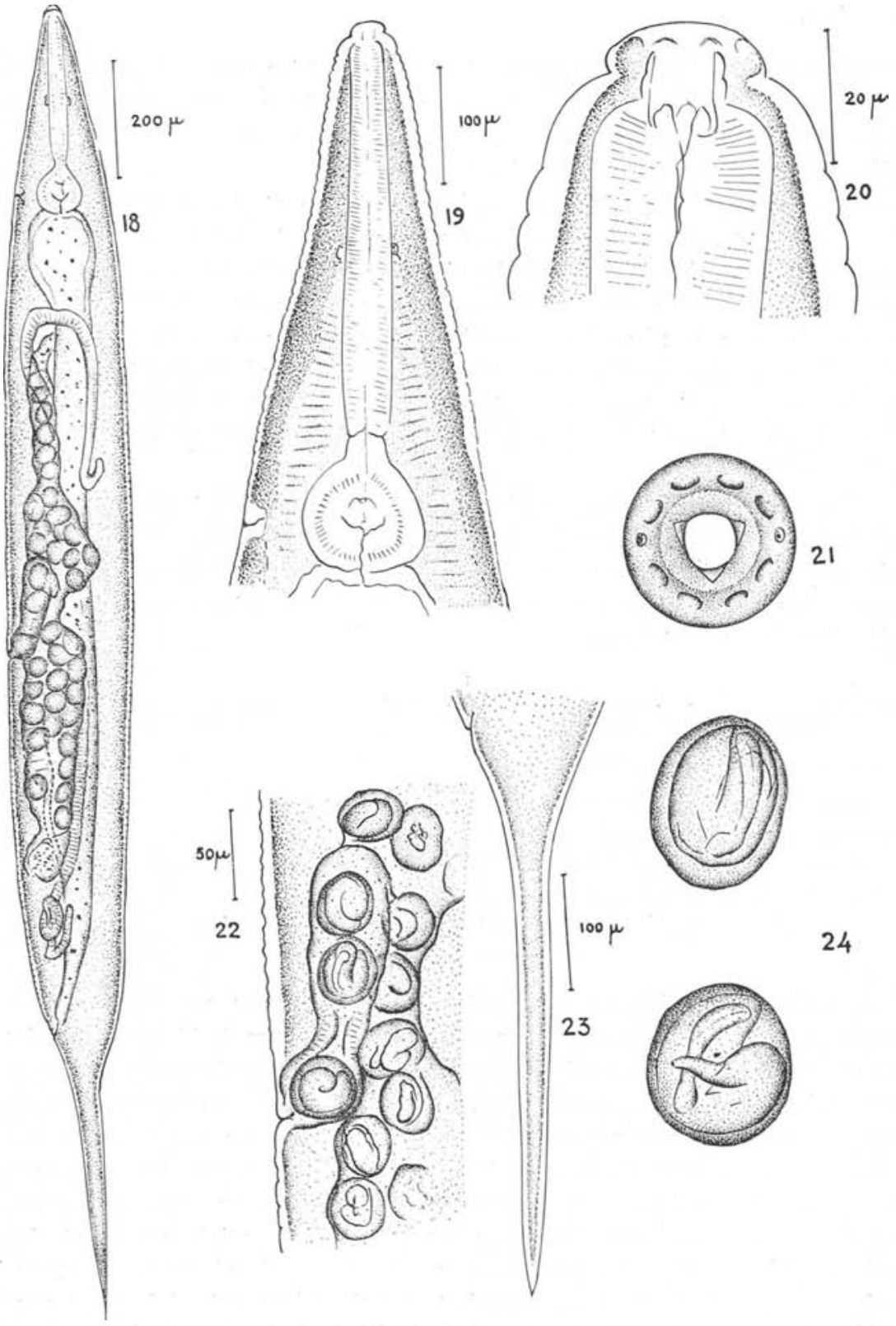

PL. IV. - Thelastoma madecassa n. sp.

A) La femelle : 18. Vue d'ensemble; 19. L'œsophage; 20. La tête, vue latérale ; 21 . La tête, vue apicale; 22. La vulve; 23 . La queue; 24 . Les œufs. 


\section{Discussion.}

Les genres Thelastoma et Cephalobellus sont voisins; les clefs de détermination (Basir 1956) utilisent pour les distinguer la forme de la queue, longue et filiforme dans le genre Thelastoma, élargie et plus courte cans le genre Cephalobellus. L'espèce étudiée entrerait donc dans le deuxième genre bien que la queue de la femelle soit relativement longue. Mais chez les espèces du genre Cephalobellus, l'ouverture du pore excréteur est nettement en arrière du bulbe, ce qui n'est pas le cas ici. En fait, l'espèce la plus proche de celle décrite ci-dessus est Bulhoesia magalhaesi (forme de la tête et de la queue analogues, début de segmentation dans l'utérus, mais position différente du pore excréteur, segmentation des œufs plus avancée chez Thelastoma madecassa, etc...) espèce décrite par Schwenk en 1926, placée par les auteurs européens dans le genre Cephalobellus Cobb, 1920 (Basir, 195E et Leibersperger, 1960) et par les auteurs sud-américains dans le genre Thelastoma Leidy, 1850 [Travassos (1920) et Kloss (1965)].

Kloss (1965) ne reconnaît d'ailleurs pas le genre Cephalobellus et lui préfère le genre Scarabanema Christie, 1931, genre mis en synonymie avec Cephalobellus par ce dernier auteur (Christie, 1933). Sans nous étendre davantage sur cette situation assez confuse, nous nous baserons sur la position du pore excréteur pour placer l'espèce étudiée dans le genre Thelastoma.

$4^{\circ}$ Thelastoma pachyjuli (Parona, 1896), Travassos 1929 var tampoketsii nov.

DESCRIPTION DE LA FEMELLE :

Longueur du corps: $1910-3110 \mu$.

Largeur du corps: 146-328 $\mu ; a$ 12,5-13,5.

Longueur de l'œsophage: $402-438 \mu ; b$ 4,7-7,1.

Longueur de la queue: $531-860 \mu ; c$ 3,6-3,8.

Distance de la vulve à l'apex : 1014-1640 $\mu$; V 51,5-53,1\% .

$n: 5$.

La cuticule est striée sur toute la longueur, la distance entre chaque strie étant comprise entre $7 \mu$ et $10 \mu$ (intervalle maximum au niveau de l'isthme). La tête (diamètre $25 \mu$, hauteur $10 \mu$ ) précède un premier anneau de longueur supérieure aux suivants (diamètre $75 \mu$, longueur $12 \mu$ ). En vue apicale, l'ouverture buccale est triangulaire. Les amphides sont arrondis et les papilles céphaliques sont larges et peu proéminentes. Le stoma a un diamètre de $10 \mu$ et une profondeur de $16 \mu$. Le corpus (longueur $296 \mu$ ) est formé par un procorpus cylindrique (diamètre $23 \mu$ ) et un métacorpus renflé à ses deux extrémités (diamètre maximum $31 \mu$, minimum $27 \mu$ ). L'isthme (diamètre minimum $25 \mu$, longueur $26 \mu$ ) est nettement séparé du corpus et du bulbe (diamère $77 \mu$ ). L'anneau nerveux est à $174 \mu$ de l'apex, soit légèrement postérieur au milieu dı corpus. Le pore excréteur est situé à la limite entre le bulbe et l'intestin moyen. L'intestin moyen forme un cardia peu marqué. L'appareil reproducteur est amphidelphe. Le nombre d'œufs contenus dans l'utérus ne dépasse que rarement 20. 

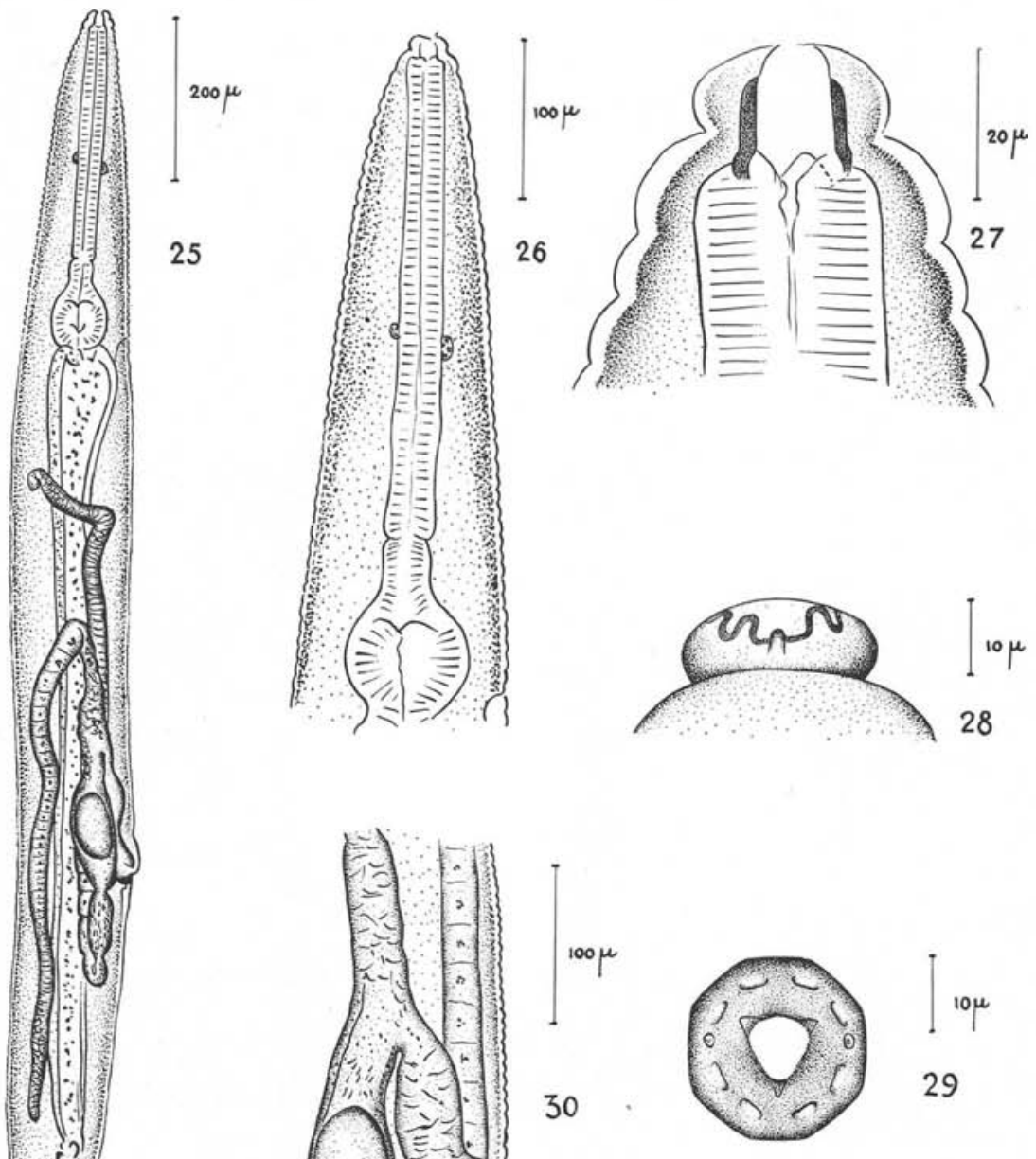

PL. V et VI. - Thelastoma pachyjuli tampoketsii var. nov.

A) La femelle: 25. Vue d'ensemble; 26. L'œsophage ; 27. La tête, coupe longitudinale; 28. La tête, vue latérale ; 29 . La tête, vue apicale ; 30 . La vulve. 


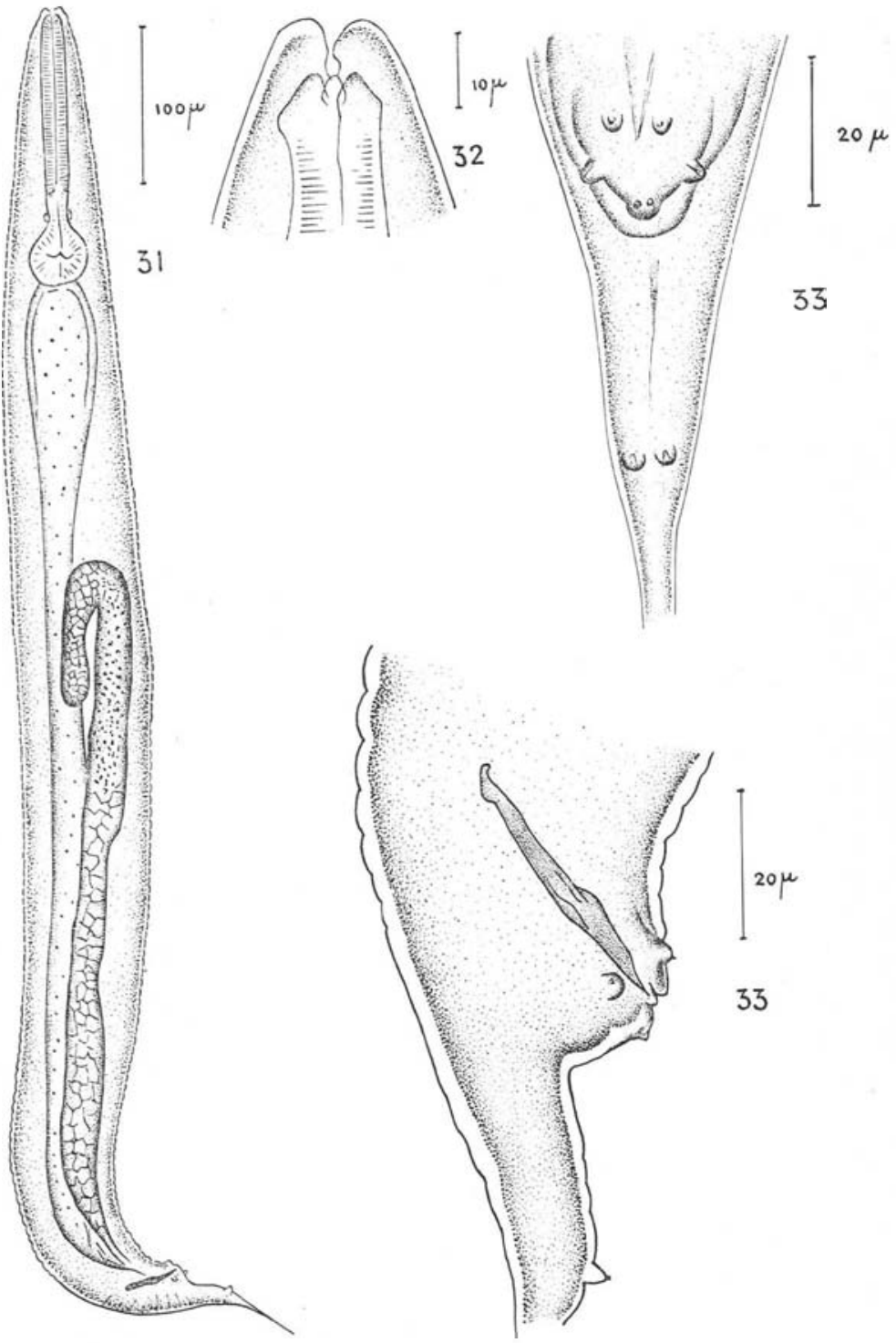

B) Le mâle : 31 . Vue d'ensemble ; 32. La tête ; 33. Le cône génital : vue latérale et vue ventrale.

La vulve est située à 71-73\% de la longueur du corps (moins la queue). Les œufs ont une forme ellipsoïdale, avec un diamètre maximum de 75 à $77 \mu$ et minimum de 58 à $60 \mu$. La queue est filiforme et a une longueur un peu supérieure au quart de la longueur totale du corps. 
LE MÂLE :

Longueur du corps : $935-1091 \mu$.

Largeur du corps : $80-85 \mu ; a 11,5-12,9$.

Longueur de l'œsophage: 169-172 $\mu ; b$ 5,5-6,4.

Longueur de la queue: $91-108 \mu ; c$ 10,1-10,7.

$n: 4$.

La cuticule est finement striée, les stries n'étant bien visibles que dans la partie postérieure du corps $(6 \mu$ entre chaque strie.) Le corpus (diamètre $16 \mu$, longueur $115 \mu$ ) n'est pas nettement séparé de l'isthme (diamètre minimum $8 \mu$, longueur $19,5 \mu$ ). Le bulbe a un diamètre de $35 \mu$. L'anneau nerveux est situé à la base de l'isthme et le pore excréteur est postérieur au bulbe (à $26 \mu$ environ). L'intestin moyen forme un cardia peu marqué en arrière du bulbe. Le spicule a une longueur de $36 \mu$ et une largeur de $3 \mu$. La protubérance préanale est peu saillante et porte trois paires de papilles (une préanale, une latérale, une postnale). Une quatrième paire est située aux deux cinquièmes de la distance de l'anus à l'extrémité caudale. La queue est filiforme et de longueur inférieure au $1 / 10^{\circ}$ de la longueur totale du corps.

Hôte: Blatta sp. (Intestin postérieur).

Localité: Tampoketsa d'Ankazobe.

Ce Nématode a toujours été trouvé en très petit nombre dans chaque hôte.

Diagnose. Femelle: pore excréteur situé à la limite entre le bulbe et l'intestin moyen. Vulve au-delà des $7 / 10^{\circ}$ de la distance de l'apex à l'anus. Eufs non ornementés. Mâle: protubérance anale développée. Longueur de la queue inférieure au $1 / 10^{\circ}$ de la longueur du corps.

\section{Discussion.}

Malgré les efforts de Basir (1956) et Kloss (1965) la systématique du genre Thelastoma reste assez confuse et les caractères permettant de distinguer les espèces sont peu sûrs. Deux espèces ressemblent à Thelastoma pachyjuli.

$1^{\circ}$ Les œufs de Thelastoma riveroi Chitwood, 1932, sont ornés de' striations. Mais Kloss 1965 met Thelastoma riveroi en synonymie avec Thelastoma attenuatum Leidy. 1849 , dont les œufs ne sont pas ornementés, et qui diffère par d'autres caractères tels que la forme de la queue du mâle, longue et filiforme chez le mâle de Thelastoma attenuatum décrit par Kloss (longueur supérieure au quart de la longueur totale du corps) plus courte (un septième de la longueur du corps) et avec une base plus large chez Thelastoma riveroi. Aussi, nous pensons qu'il est préférable de conserver cette dernière espèce qui se distingue donc de $T$. pachyjuli par l'ornementation des œufs et d'autres caractères secondaires (queue du mâle relativement plus longue, position du pore excréteur en face du bulbe, œsophage de la femelle relativement plus long, etc...).

$2^{\circ}$ En 1960, Leibersperger décrit Thelastoma rovinjense. Kloss (1966) met cette espèce en synonymie avec Thelastoma magalhaesi (Schwenk 1926) espèce classée par 

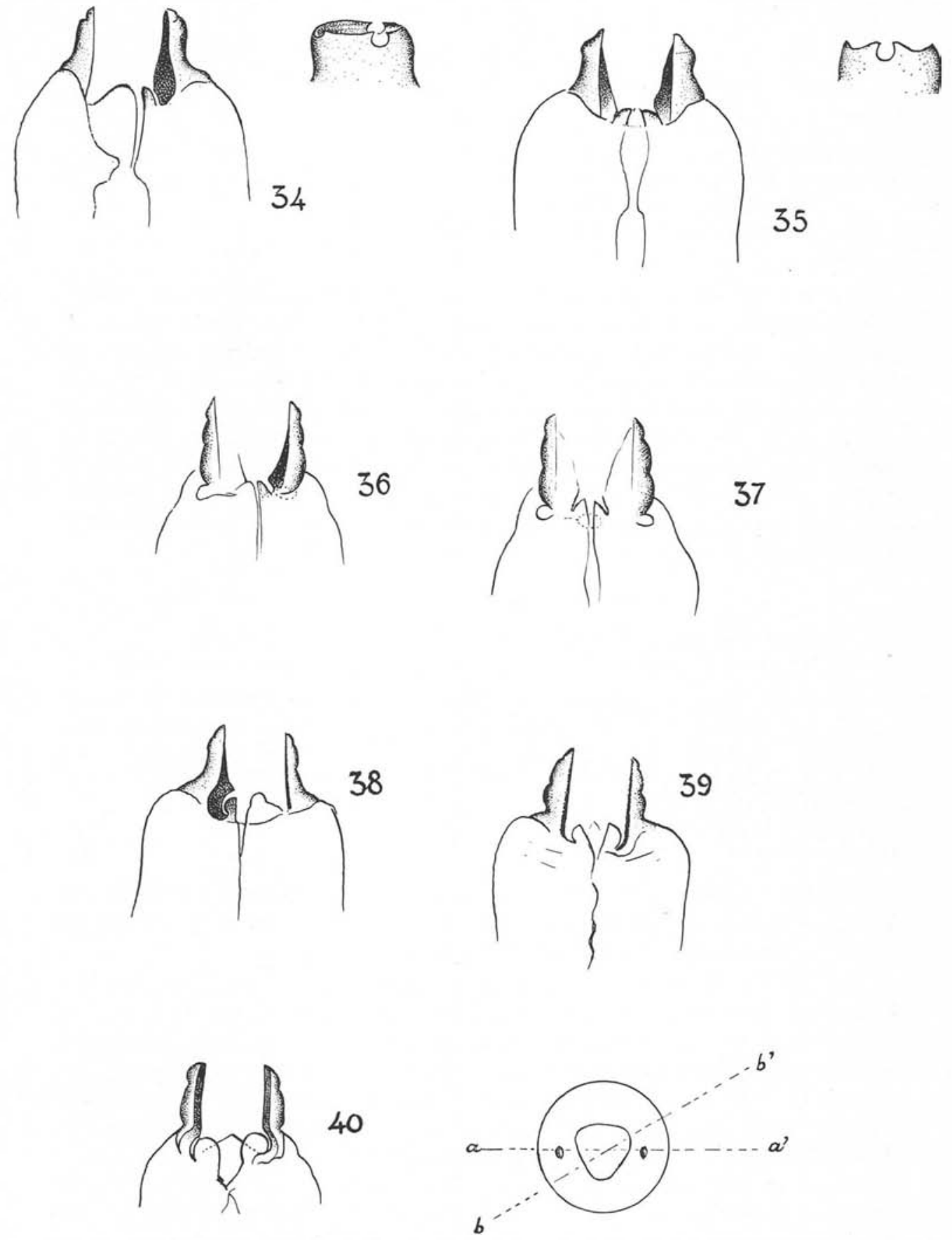

PL. VII. - La structure du stoma

34. Protrellus rasolofi: coupe suivant bb' et vue externe du stoma ; 35 . Protrellus rasolofi: coupe suivant aa' et vue externe du stoma ; 36 . Protrellus behorefi : coupe suivant b'b ; 37 . Protrellus behorefi : coupe suivant aa'; 38. Thelastoma madecassa: coupe suivant bb'; 39 . Thelastoma madecassa : coupe suivant aa'; 40 . Thelastoma pachyjuli tampoketsii : coupe suivant aa'. 
Leibersperger dans le genre Céphalobellus. T. pachyjuli tampoketsii se distingue de cette dernière espèce par la taille deux fois plus petite de la femelle, la queue de la femelle rélativement plus longue (inférieure au quart de la longueur du corps), la queue du mâle plus courte, la forme de la protubérance anale (nettement moins saillante).

$3^{\circ}$ Il n'y a pas de différence fondamentale entre Thelastoma pachyjuli (Parona 1896) Travassos 1929 et la forme que nous avons décrite. La position du pore excréteur, utilisée par Leibersperger dans la clef des espèces, n'est pas la même (en avant du bulbe chez Thelastoma pachyjuli). Les proportions sont quelquefois différentes (œsophage du mâle relativement plus petit, position de l'anneau nerveux plus en retrait chez cette dernière espèce). Aussi, nous pensons que nous sommes en présence d'une forme malgache de cette espèce cosmopolite (déjà trouvée en Amérique du Nord et du Sud, et en Europe sur des Blattes et des Diplopodes.

Les spécimens types décrits ci-dessus sont déposés au Museum National d'Histoire Naturelle, à Paris. Les paratypes sont déposés au centre ORSTOM à Tananarive.

\section{Bibliographie}

BASIR (M. A.), 1956. - Oxyuroid parasites of arthropoda. A monographic study. 1. Thelastomatidae. 2. Oxyuridae. Zoologica, Stuttgart, 38 (108), 79 p., 13 pl.

CHITwood (B. G.), 1933. - A synopsis of the nematodes parasitic in insects of the family Blattidae. Zeitschr. P. Parasitenkde, Jena, 5 (1): 14-50, 59 figs.

Christie (J. R.), 1933. - The generic names Cephalobellus Совв 1920 and Scarabonema, Christie 1931. J. Wash. Acad. Sci., 23, 358.

Dale (P. S.), 1966. - Blatticola tuapakae and Protrellina gurri n. spp., Nematode parasites of the Black Roach. N.Z. Jl. Sci., 9, 538-544.

KLoss (G. R.), 1961. - Dois nematodes parasitos intestinais de especies selvagens de Blattaria. Papeis Avulsos Dep. Zool. S. Paulo, 14, (26), 243-247.

-, 1965. - Consideraçoes en torno de Thelastoma, LeIDy 1850 e Cephalobellus, CовB 1920 (nematoda). Papeis Avulsos Dep. Zool. S. Paulo, 17, (13), 131-179, 56 fig.

-, 1966. - Revisao dos nematoides de Blattaria do Brasil. Papeis Avulsos Dep. Zool. S. Paulo, 18, (16), 147-188.

Leibersperger (E.), 1960. - Oxyuroidae der europaischen Arthropoden. Parasit. Schrift., 11, 150 p., 39 fig. 